\title{
Preclinical exploration of combining plasmacytoid and myeloid dendritic cell vaccination with BRAF inhibition
}

\author{
Jurjen Tel ${ }^{1}$, Rutger Koornstra ${ }^{2 \dagger}$, Nienke de Haas ${ }^{1 \dagger}$, Vincent van Deutekom ${ }^{1}$, Harm Westdorp ${ }^{1,2}$, \\ Steve Boudewijns ${ }^{1,2}$, Nielka van Erp³, Stefania Di Blasio', Winald Gerritsen², Carl G. Figdor?', \\ I. Jolanda M. de Vries ${ }^{1 *}$ and Stanleyson V. Hato ${ }^{1}$
}

\begin{abstract}
Background: Melanoma is the most lethal type of skin cancer and its incidence is progressively increasing. The introductions of immunotherapy and targeted therapies have tremendously improved the treatment of melanoma. Selective inhibition of BRAF by vemurafenib results in objective clinical responses in around $50 \%$ of patients suffering from BRAFV600 mutated melanoma. However, drug resistance often results in hampering long-term tumor control. Alternatively, immunotherapy by vaccination with natural dendritic cells (nDCs) demonstrated long-term tumor control in a proportion of patients. We postulate that the rapid tumor debulking by vemurafenib can synergize the long-term tumor control of nDC vaccination to result in an effective treatment modality in a large proportion of patients. Here, we investigated the feasibility of this combination by analyzing the effect of vemurafenib on the functionality of nDCs.

Methods: Plasmacytoid DCs (pDCs) and myeloid DCs (mDCs) were isolated from PBMCs obtained from buffy coats from healthy volunteers or vemurafenib-treated melanoma patients. Maturation of pDCs, mDCs and immature monocyte-derived DCs was induced by R848 in the presence or absence of vemurafenib and analyzed by FACS. Cytokine production and T cell proliferation induced by mature DCs were analyzed.

Results: Vemurafenib inhibited maturation and cytokine production of highly purified nDCs of healthy volunteers resulting in diminished allogeneic T cell proliferation. This deleterious effect of vemurafenib on nDC functionality was absent when total PBMCs were exposed to vemurafenib. In patients receiving vemurafenib, nDC functionality and T cell allostimulatory capacity were unaffected.

Conclusion: Although vemurafenib inhibited the functionality of purified nDC of healthy volunteers, this effect was not observed when nDCs were matured in the complete PBMC fraction. This might have been caused by increased vemurafenib uptake in absence of other cell types. In accordance, nDCs isolated from patients on active vemurafenib treatment showed no negative effects. In conclusion, our results pave the way for a combinatorial treatment strategy and, we propose that combining vemurafenib with $\mathrm{nDC}$ vaccination represent a powerful opportunity that deserves more investigation in the clinic.
\end{abstract}

Keywords: Vemurafenib, DC vaccination, Plasmacytoid DCs, BDCA1 + myeloid DCs

\footnotetext{
*Correspondence: jolanda.devries@radboudumc.nl

${ }^{\dagger}$ Rutger Koornstra and Nienke de Haas equally contributed to this work

${ }^{1}$ Department of Tumor Immunology, Radboud Institute for Molecular Life

Sciences, Radboud University Medical Center, 6500 HB Nijmegen,

The Netherlands

Full list of author information is available at the end of the article
} 


\section{Background}

Melanoma is a highly malignant melanocyte-derived tumor with a rising incidence. It is the most lethal of all skin cancers, accounting for $80 \%$ of all skin cancer related deaths while representing only $4 \%$ of all cases [1]. Recently, advances in both targeted therapies, as well as, immunotherapy have finally begun to show effectiveness against metastatic melanoma [2-7]. Mutations in the BRAF signaling pathway have been identified as one of the drivers of melanoma. Mutations in the BRAF gene can be detected in 50-60\% of cutaneous melanomas and mutations in the NRAS gene in $10-20 \%[8,9]$. These mutations lead to constitutive activation of the mitogen activated protein kinase (MAPK) pathway, which in turn, results in increased cell proliferation and survival [10, 11]. Targeted agents directed against the MAPK pathway, mostly small molecule inhibitors, such as vemurafenib, have recently demonstrated clinical efficacy in metastatic disease $[2,3,12]$. Vemurafenib is a selective inhibitor of mutated BRAF and shows great efficacy in metastatic melanoma patients harboring the BRAFV600 mutation $[2,3,12]$. Objective clinical responses are seen in around $50 \%$ of all patients and the drug is generally well tolerated $[2,3,12]$. Despite the rapid responses and high response rates, drug resistance develops in the vast majority of patients, which clearly is an obstacle for achieving longterm tumor control. Even more, it has been reported that tumors might become dependent on vemurafenib, which has led to the postulation of intermitted treatment schedule in order to prevent resistance [13].

The other novel treatment modality for melanoma, which even was designated as breakthrough of the year 2013 [14], is the use of immunotherapy. Broadly immunotherapy can be divided in cellular immunotherapy, such as dendritic cell (DC) vaccination, and antibody-based immunotherapy, such as immune check point blockade [15]. DCs are professional antigen presenting cells that can be loaded with melanoma-associated antigens and used to mount an immune response against tumor cells by activating cytotoxic $\mathrm{T}$ cells. DC vaccination for melanoma has been explored in many phase I and II studies. Thus far, virtually all of these clinical studies have been performed with ex vivo differentiated monocyte-derived DCs (moDCs) or from CD34 ${ }^{+}$progenitors. Although numerous vaccination studies demonstrated the immunogenicity of tumor antigen-loaded DCs, the number of objective clinical responses has been limited, hampering its implementation as a novel form of standard treatment. However, DC vaccination still has untapped potential as, some stage IV melanoma patients did experience long lasting clinical responses. Additionally, in stage III melanoma there was a favorable overall survival benefit after adjuvant DC vaccination [16]. Additionally, recent trials exploiting naturally occurring DC subsets (nDCs), which circulate in the blood, as a vaccine vehicle showed promising increases in overall survival $[17,18]$.

Another immunotherapeutic strategy, the blocking of CTLA-4 with ipilimumab led to enhanced T cell activation and increased tumor rejection $[4,19]$ and resulted in long-term tumor control in about $20 \%$ of patients. As number of studies showed that BRAF inhibition increased the number of intratumoral cytotoxic $\mathrm{T}$ cells and increased expression of tumor-associated antigens on tumor cells [20-23], it was hypothesized that BRAF inhibition could synergize with CTLA-4 blockade. Unfortunately, the first trial to study combination of ipilimumab and vemurafenib was terminated prematurely due to severe hepatotoxicity [24]. This study showed that the combination of vemurafenib with checkpoint inhibition is not feasible, at least not when given simultaneously. Recently, other studies investigated the potential of combinations of more BRAF inhibitors, like dabrafenib, and checkpoint inhibition (ipilimumab) with or without additional treatment with the MEK inhibitor trametinib (NCT01940809 and NCT02130466). Preliminary results indicate that these combinations are tolerated, and do not result in severe hepatotoxicity. Nevertheless, the combination of three agents was discontinued based on the development of colitis followed by intestinal perforation [25-27]. We have recently shown that using natural blood-borne DC subsets as vehicles for DC vaccination, markedly improved responses in terms of overall survival could be observed, with minimal toxicity $[17,28]$. With that in mind, we postulate that $\mathrm{DC}$ vaccination might be a candidate to be combined with vemurafenib. In this preclinical study, we investigated the feasibility of combining $D C$ vaccination with vemurafenib by studying whether modulation of MAPK signaling pathway affected function and maturation of $\mathrm{nDCs}$ in healthy volunteers or melanoma patients receiving vemurafenib.

\section{Methods \\ Cells}

Isolation of nDCs was described previously [29]. Briefly, buffy coats were obtained from healthy volunteers with informed consent according to institutional and international guidelines. Blood from end-stage metastatic melanoma patients was collected prior to start of vemurafenib treatment and after 1 month vemurafenib therapy. This study was approved by the local Institutional Review Board (Committee on Research involving Human Subjects Arnhem-Nijmegen) and in accordance with the declaration of Helsinki. Written informed consent was obtained from all patients. After peripheral blood mononuclear cells (PBMC) isolation, 
plasmacytoid DCs (pDCs) were purified by positive isolation using anti-BDCA-4-conjugated magnetic microbeads, and BDCA- $1^{+}$myeloid DCs (mDCs) were purified using anti-CD1c-conjugated microbeads (both Miltenyi Biotec, Bergisch-Gladbach, Germany) after $\mathrm{B}$ cell depletion. Plasmacytoid $\mathrm{DC}$ and $\mathrm{mDC}$ purity was routinely up to $95 \%$, as assessed by double staining with BDCA-2/CD123 or CD11c/CD1c (all Miltenyi Biotec). DCs were cultured in X-VIVO-15 (Lonza, Verviers, Belgium) supplemented with $10 \%$ human serum (Sanquin, Nijmegen, the Netherlands). DC maturation was induced through addition of $4 \mu \mathrm{g} / \mathrm{ml} \mathrm{R} 848$ (Axxora, San Diego, CA). Monocyte-derived DCs (moDCs) were generated from adherent PBMCs, by culturing in the presence of IL-4 $(500 \mathrm{U} / \mathrm{ml})$ and GM-CSF $(800 \mathrm{U} /$ ml) (both Cellgenix, Freiburg, Germany). Cells were cultured in X-VIVO 15 medium supplemented with $2 \%$ human serum and harvested on day 6 as immature DC. Immature $\mathrm{DC}$ or $\mathrm{mDCs}$ were activated through the addition of $4 \mu \mathrm{g} / \mathrm{ml} \mathrm{R} 848$ and/or $20 \mu \mathrm{g} / \mathrm{ml}$ Poly I:C (Sigma-Aldrich).

\section{Flow cytometry}

Purity of pDCs and mDCs after isolation and the phenotype of the pDC populations were determined by flow cytometry. The following primary monoclonal antibodies (mAbs) and the appropriate isotype controls were used: anti-BDCA1-FITC, BDCA2-PE, BDCA4-PE and CD123APC (all Miltenyi Biotec); mIgG1-PE, mIgG1-APC, antiCD11c-FITC or -APC, anti-HLA-ABC-PE (W6/32), anti-CD80-PE, or -APC, or -PeCy7, anti-CD86-PE, or -APC (all BD Bioscience Pharmingen, San Diego, CA, USA) anti-PD-L1-APC, anti-PD-L2-PE; anti-CD40-PE, anti-CD83-PE (Beckman Coulter, Mijdrecht, the Netherlands); anti-MHC-II-APC (eBioscience).

The phenotype of the DC populations after treatment with vemurafenib, dabrafenib, trametinib or a combination was determined by staining with the following antibodies and appropriate isotype controls: anti-CD80-PE-Cy7, anti-CD86 APC, anti-PD-L1-PE, anti-HLA-ABC-V450, anti HLA-DR-BV510, mIgG1-PECy7, mIgG1-PE (all BD biosciences) and mIgG1-APC (eBioscience).

\section{Reagents}

Vemurafenib (PLX4032 or RG7204) was kindly donated by Roche for study purpose. Vemurafenib was dissolved in DMSO to a stock concentration of $1 \mathrm{mg} / \mathrm{ml}$. The vemurafenib stock was freshly diluted before each experiment to $60 \mu \mathrm{g} / \mathrm{ml}$ unless stated otherwise. Dabrafenib and trametinib were obtained from Alsachim (IllkirchGraffenstaden, France). Both drugs were diluted freshly to 90 and $12 \mathrm{ng} / \mathrm{ml}$, respectively.

\section{Antigen-specific $\mathrm{T}$ cell activation}

pDCs and mDCs from a HLA-A2.1 + donor were loaded with different concentrations of a melanoma-specific peptide $\left(\mathrm{gp}^{100} \mathrm{0}_{280: 288}\right)$ or irrelevant peptide (tyrosinase $\left._{369: 376}\right)$ in 96-well round bottom plates $\left(10 \times 10^{3}\right.$ cells per well). After approximately 2 h, R848, two concentrations of vemurafenib and gp100 $280: 288^{-s p e c i f i c ~} \mathrm{~T}$ cells $\left(50 \times 10^{3}\right.$ cells per well) were added. After overnight incubation, CD69 and CD25 expression on the CD3 ${ }^{+}$ gp $100_{280: 288^{-}}$-specific $\mathrm{T}$ cells was measured by flow cytometry using PE-Cy5 conjugated mouse anti-human CD69, APC-conjugated CD25 and BV421-conjugated CD3 (all BD biosciences).

\section{Mixed lymphocyte reaction}

Allogeneic peripheral blood lymphocytes (PBLs) were co-cultured with differently matured $\mathrm{pDCs}, \mathrm{mDCs}$ and moDCs in a 96-well round-bottom plate $\mathrm{pDC} / \mathrm{PBL}$ ratio 1:20 with $10^{5}$ PBLs). After 3 days of culture at $37{ }^{\circ} \mathrm{C}$, $1 \mu \mathrm{Ci} /$ well ([0.037 MBq]/well; MP Biomedicals, Amsterdam, the Netherlands) of $\left[{ }^{3} \mathrm{H}\right]$-thymidine was added for $12 \mathrm{~h}$ and incorporation was measured in a beta-counter.

\section{Cytokine detection}

pDCs and mDCs were cultured overnight at a concentration of $10^{5} \mathrm{DCs} / 100 \mu \mathrm{l} /$ well in a 96-well round-bottom (pDCs) or flat-bottom (mDC) plate. Supernatants were collected from DC cultures after $16 \mathrm{~h}$ of activation; IL-6, TNF $\alpha$, RANTES, IP-10 and MIP- $1 \alpha$ production was measured using a human Multiplex kit (BMS817FF from eBioscience) according to manufacturer's instructions. IFN $\alpha$ production was measured by ELISA according to manufacturer's instructions.

\section{Statistics}

All experiments were performed at least three times and results are shown as the mean \pm SEM. Data sets were either tested by a Student's $t$ test or by one-way ANOVA followed by Newman-Keuls or Dunnett's multiple comparison test.

\section{Results}

At steady-state blood concentration vemurafenib inhibits nDC maturation and cytokine secretion in vitro

We explored whether vemurafenib had any effect on the viability, phenotype or maturation of the $\mathrm{nDC}$ subsets, pDCs and mDCs. Both DC subsets were exposed to $60 \mu \mathrm{g} / \mathrm{ml}$ of vemurafenib, which is higher than in most other studies and higher than the IC50 for most tumor cells in vitro [30]. However, $60 \mu \mathrm{g} / \mathrm{ml}$ is the average steady-state concentration $\left(C_{\text {trough }} ; 34-93 \mu \mathrm{g} / \mathrm{ml}\right)$ of vemurafenib in the blood of patients treated with vemurafenib determined in pharmacokinetic studies [3, 31]. 
As such, this is the concentration of vemurafenib that circulating nDCs will be exposed to. This concentration of vemurafenib had deleterious effects on $\mathrm{nDC}$ viability cultured in medium containing $2 \%$ human serum (Fig. 1; Additional file 1: Fig. S1). Taking into account that $>99 \%$ of vemurafenib in the blood is protein bound and the low amount of serum (and protein) used here, we investigated the effect of increasing the amount of serum to 5-10\%. DC viability was improved with both concentrations and $10 \%$ serum restored $\mathrm{pDC}$ and $\mathrm{mDC}$ viability to the level of untreated cells (Fig. 1). In accordance with previous findings, vemurafenib had no negative effects on $\mathrm{T}$ cell and $\mathrm{B}$ cell viability (Additional file 2: Fig. S2) [20]. Additionally, there was no effect on the viability of pDCs and mDCs matured in the presence of vemurafenib at a concentration of $10 \%$ human serum (Fig. 1). The next step was to investigate whether exposure to vemurafenib during maturation at a concentration of $10 \%$ human serum had any effect on the resulting phenotype. The single stranded RNA analog R848 (agonist for TLR$7 / 8$ ) was used to trigger DC maturation as it stimulates both mDCs and pDCs. R848 induces CD40, CD80 and CD86 indicative for maturation of both pDCs and mDCs (Fig. 2a, b). However, exposure to vemurafenib clearly hampered the maturation of these blood DC subsets as all markers failed to reach similar expression levels as cells cultured without vemurafenib.

Next, we determined whether vemurafenib could also modulate TLR-induced proinflammatory cytokine and chemokine secretion by pDCs and mDCs. We observed a marked decrease in the secretion of IL-6, TNF-a, and IP-10 by R848 stimulated pDCs and mDCs (Fig. 2c, d). Additionally, vemurafenib exposure also inhibited interferon- $\alpha$ (IFN- $\alpha$ ) production by pDCs.

In order to investigate whether these observed effects were exclusively vemurafenib-related or class-specific for BRAF inhibition, we performed similar experiments with the BRAF inhibitor dabrafenib. Furthermore, we extended our analysis by studying the effects of the MEK inhibitor trametinib and the different combinations of these inhibitors. Importantly, the $\mathrm{IC}_{50}$ values for both dabrafenib and trametinib are approximately 40 times lower compared to vemurafenib under cell-free assay conditions. In vivo, this translates into much lower levels compared to vemurafenib, the $C_{\text {through }}$ values for dabrafenib and trametinib are 90 and $12 \mathrm{ng} / \mathrm{ml}$, respectively [32, 33], compared to $60 \mu \mathrm{g} / \mathrm{ml}$ for vemurafenib. We cultured $\mathrm{mDCs}$ and $\mathrm{pDCs}$ with the drugs either alone or in various combinations in the presence of R848. After overnight activation we observed that while vemurafenib inhibited the R848 induced-maturation of both pDCs and $\mathrm{mDCs}$, neither dabrafenib nor trametinib had a significant impact on the upregulation of CD80, CD86, PD-L1, MHC-I or MHC-II (Fig. 3a, b). Similarly, the combination of vemurafenib and trametinib inhibited maturation just like vemurafenib while the combination of dabrafenib and trametinib did not (Fig. 3a, b). Taken together, vemurafenib exerts negative off-target effects on both $\mathrm{mDCs}$ and $\mathrm{pDCs}$ obtained from healthy donors, while other small molecule inhibitors with higher specificity and selectivity do not hinder $\mathrm{mDC}$ or $\mathrm{pDC}$ maturation in vitro.

\section{Exposure to vemurafenib leads to downregulation of MHC molecules, decreased allostimulatory capacity, and minimally decreased antigen-specific $\mathrm{T}$ cell activation} The expression levels of MHC class I, and MHC class II, which are necessary to activate $\mathrm{CD} 8^{+}$cytotoxic $\mathrm{T}$ cells, and $\mathrm{CD}_{4}^{+}$helper $\mathrm{T}$ cells, respectively was also measured. Although cytotoxic T cells are the most important mediators of anti-tumor immune responses, induction of CD4 $\mathrm{T}$ cells improves clinical outcome, probably by stimulating $\mathrm{B}$ cell function [34, 35]. In addition to decreased maturation of $\mathrm{pDCs}$ and $\mathrm{mDCs}$, maturation-induced upregulation of MHC class I and MHC class II was also diminished after exposure to vemurafenib, indicating a
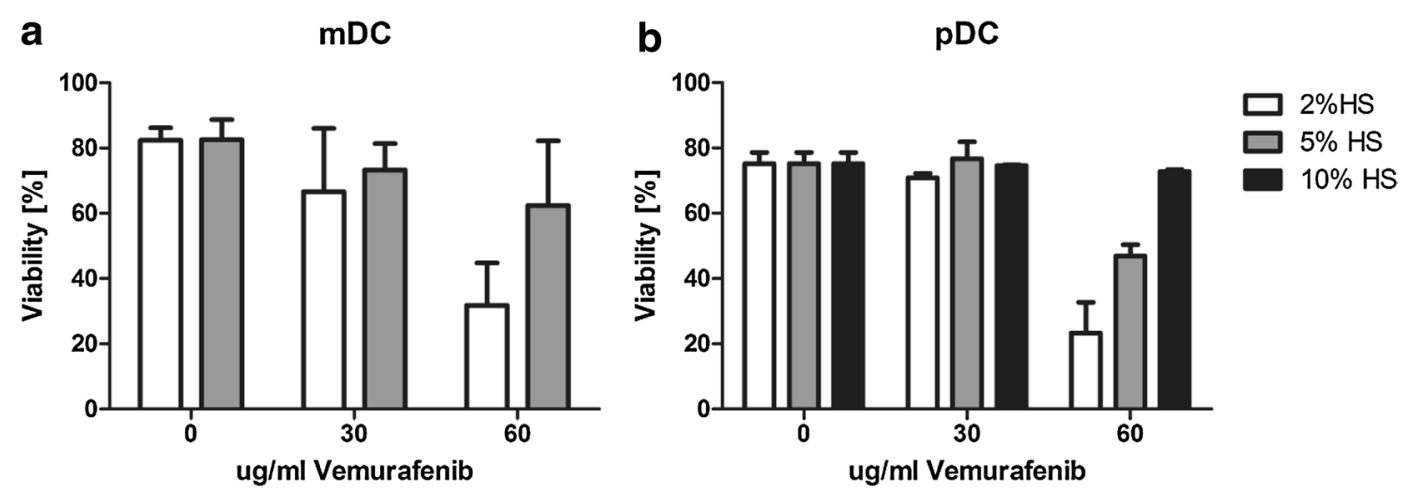

Fig. 1 Vemurafenib bioavailability determines survival of pDCs and mDCs. Freshly isolated plasmacytoid DCs and myeloid DCs were cultured in increasing concentrations of vemurafenib and human serum and cell viability was determined after $24 \mathrm{~h}$ by FACS analysis 

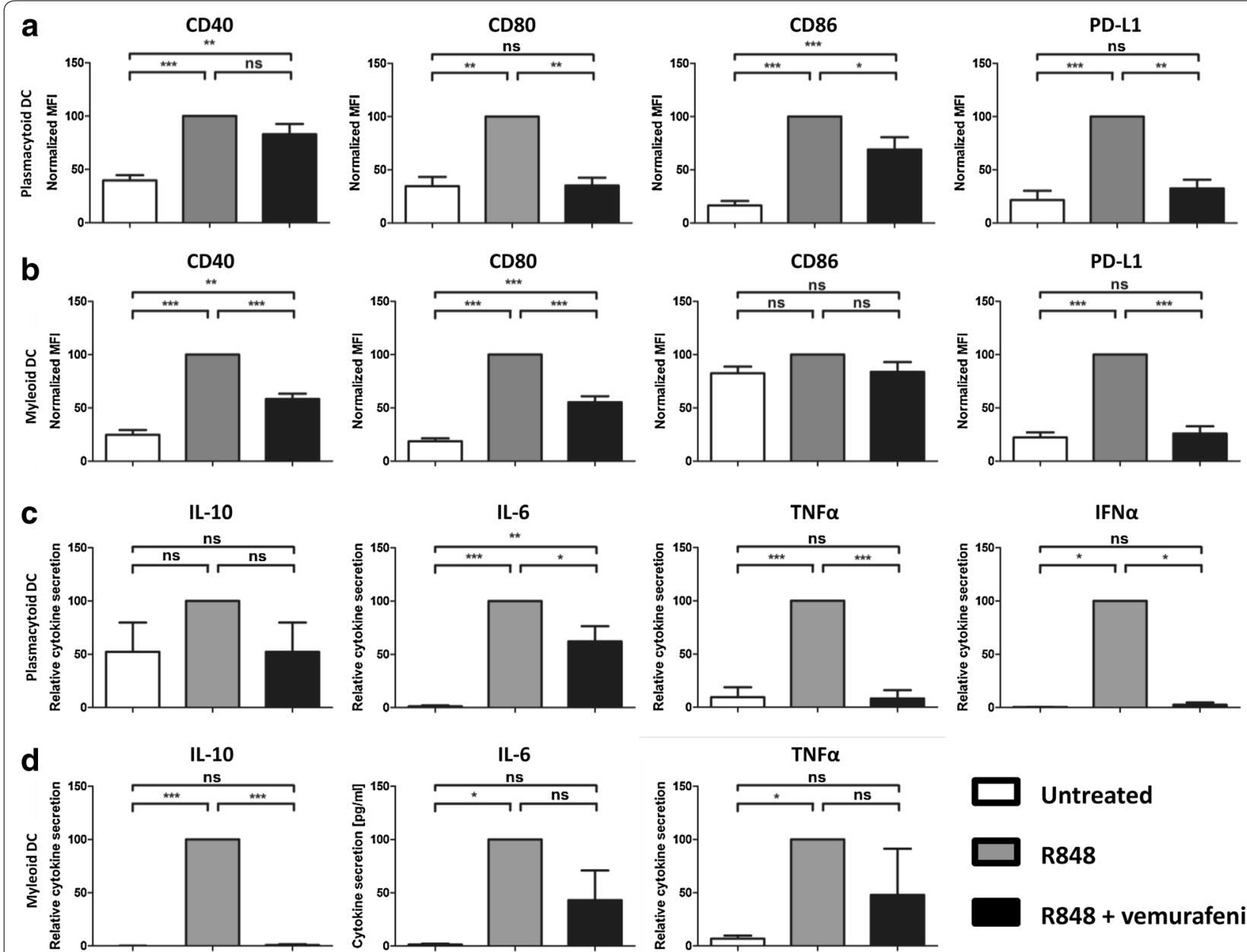

Fig. 2 Vemurafenib impairs maturation of ex vivo cultured pDCs and mDCs. Freshly isolated pDCs (a) and mDCs (b) were cultured ex vivo and activated with R848 in presence or absence of $60 \mu \mathrm{g} / \mathrm{ml}$ vemurafenib. Graphs show the cell surface expression levels of CD80, CD86, CD40, and PD-L1 after $18 \mathrm{~h}$. Freshly isolated pDCs (c) and mDCs (d) were cultured ex vivo and activated with R848 in presence or absence of $60 \mu \mathrm{g} / \mathrm{ml}$ vemurafenib. Graphs show the levels of the cytokines IL-10, IL-6, TNFa and IFNa (only pDCs) measured in the supernatant after $18 \mathrm{~h}$. Shown is the mean (+SEM) of six independent experiments ${ }^{*} P<0.05,{ }^{* * P}<0.01,{ }^{* * *} P<0.001$

possible decreased capacity of antigen presentation to $\mathrm{T}$ cells (Fig. 4a, b). Concomitantly, there was a significant decrease in allogeneic $\mathrm{T}$ cell proliferation induced by pDCs and mDCs matured in the presence of vemurafenib (Fig. 4c, d). The observed decrease in proliferation could not be due to increased expression of co-inhibitory molecules after DC stimulation in the presence of vemurafenib because PD-L2 was not expressed by either subset (data not shown) and PD-L1 upregulation was completely absent (Fig. 2a, b). Thus although vemurafenib inhibits the upregulation of PD-L1 the proliferation of allogeneic $\mathrm{T}$ cells is not enhanced but decreased, most likely due to the reduced expression of $\mathrm{MHC}$ molecules after stimulation of DC in the presence of vemurafenib. Furthermore, we examined whether vemurafenib affects the ability of pDCs and mDCs to prime antigen-specific $\mathrm{T}$ cells. For this we made use of Jurkat $\mathrm{T}$ cells that are transfected with the $\mathrm{T}$ cell receptor for gp $100_{280: 288}$. We observed that both pDCs and mDCs activated with R848 in the presence or absence of vemurafenib and loaded with various concentrations of the gp $100_{280: 288}$ peptide were able to significantly induce the activation of Jurkat $\mathrm{T}$ cells as evidenced by the specific upregulation of the IL-2 receptor CD25 (Additional file 3: Fig. S3) and the early activation marker CD69 (Fig. 4e, f). Although all conditions led to a significant activation compared to cells loaded with the irrelevant tyrosinase peptide, we observed that vemurafenib negatively affected the antigen-specific $T$ 


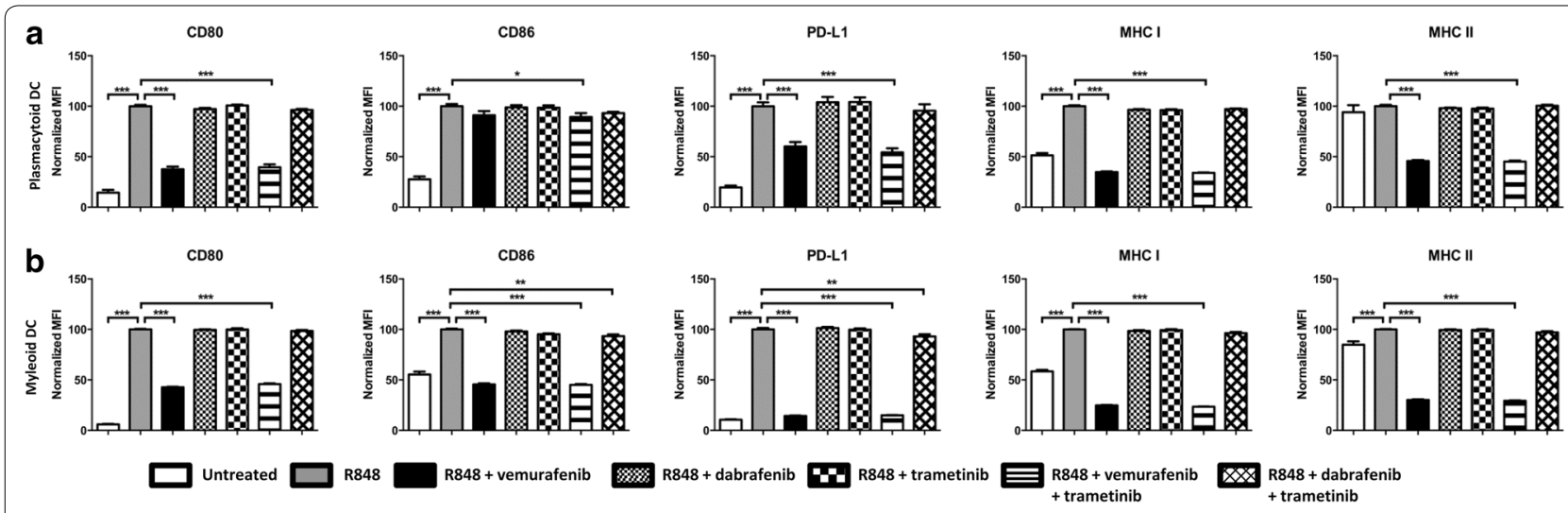

Fig. 3 Dabrafenib and trametinib do not impair maturation of ex vivo cultured pDCs and mDCs. Freshly isolated pDCs (a) and mDCs (b) were cultured ex vivo and activated with R848 in presence or absence of $60 \mu \mathrm{g} / \mathrm{ml}$ vemurafenib, $90 \mathrm{ng} / \mathrm{ml}$ dabrafenib and $12 \mathrm{ng} / \mathrm{ml}$ trametinib. Graphs show the cell surface expression levels of CD80, CD86, PD-L1, MHC-I and MHC-II after $18 \mathrm{~h}$. Shown is the mean (+SEM) of three independent experiments ${ }^{*} P<0.05,{ }^{* * P}<0.01,{ }^{* * * P}<0.001$

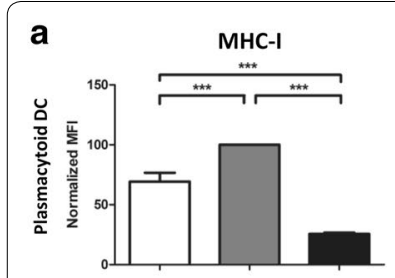

b $\mathrm{MHC-I}$

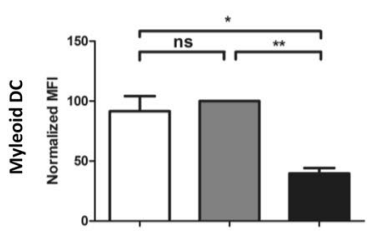

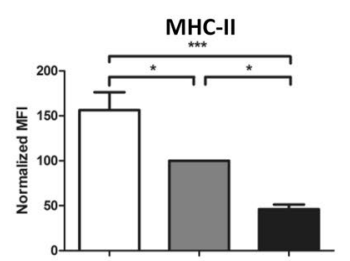

MHC-II

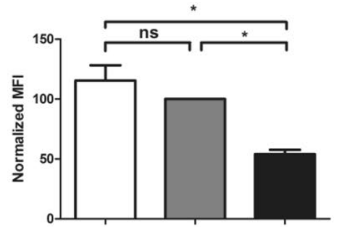

C

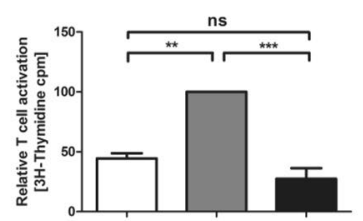

d

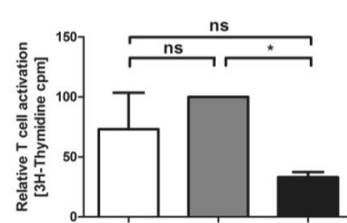

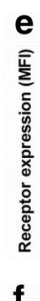

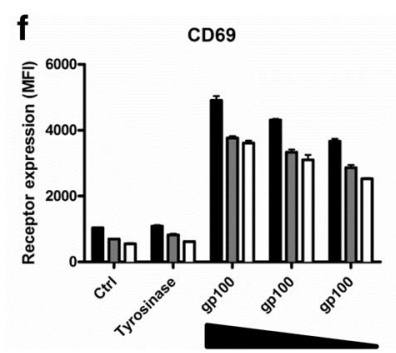

CD69

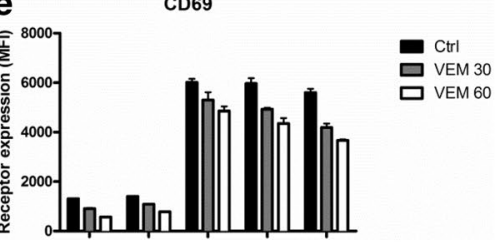

- $\mathrm{ctrl}$ 口 VEM 30 ㅁ VEM 60

Fig. 4 Vemurafenib impairs antigen presentation and allostimulatory capacity of ex vivo cultured pDCs and mDCs. Freshly isolated pDCs (a) and mDCs (b) were cultured ex vivo and activated with R848 in presence or absence of $60 \mu \mathrm{g} / \mathrm{ml}$ vemurafenib. Graphs show the cell surface expression levels of MHC class I and MHC class II after $18 \mathrm{~h}$. Mixed lymphocyte reaction using freshly isolated, pDCs (c) and mDCs (d) stimulated with R848 in presence or absence of $60 \mu \mathrm{g} / \mathrm{ml}$ vemurafenib. After maturation DCs were incubated with allogeneic PBLs and T cell proliferation was measured after 4 days with $\left[{ }^{3} \mathrm{H}\right]$ thymidine incorporation. $\mathbf{d}$ pDCs and mDCs from a HLA-A2.1 + donor were loaded with different concentrations of a melanoma-specific peptide $\left(\mathrm{gp}_{100_{280: 288}}\right.$ ) or irrelevant peptide (tyrosinase $\mathrm{S}_{369: 376}$ ) in the presence of R848 with/without vemurafenib and gp 100 $280: 288$ specific T cells. Graphs show the cell surface expression levels of the early activation marker CD69 after overnight co-culture. Shown is the mean $(+\mathrm{SEM})$ of at least three independent experiments ${ }^{*} \mathrm{P}<0.05,{ }^{* * \mathrm{P}}<0.01,{ }^{* * *} \mathrm{P}<0.001$

cell receptor signaling as the level of CD69 induction was lower under this condition. Though a clear inhibitory effect on non-antigen specific $\mathrm{T}$ cell proliferation was observed, only a minute effect of vemurafenib on antigen-specific $\mathrm{T}$ cell activation could be detected. This discrepancy might be caused by prolonged (3 days) culture of $\mathrm{T}$ cells in the presence of vemurafenib, whereas in the antigen-specific $\mathrm{T}$ cell priming the readout was after $18 \mathrm{~h}$. These findings reflect the potential in vitro toxicity of vemurafenib on immune cells. Taken together, these data imply that vemurafenib minimally decreased the antigen-specific immunostimulatory ability of blood DC subsets.

\section{The presence of immune cells mitigates the inhibitory} effects of vemurafenib on DC maturation and cytokine secretion

Previous experiments studied the effects of vemurafenib on isolated, purified $\mathrm{nDC}$ cultures. We wondered whether the presence of other immune cells during the 
exposure to vemurafenib, as would be the case in the blood stream, would have an effect on the outcome. To test this, we stimulated whole PBMC fractions of healthy volunteers with R848 in the presence or absence of vemurafenib. After $24 \mathrm{~h}$ the PBMCs were harvested and stained for DC-specific markers and maturation markers. These strategies allowed us to gate the DCs and quantify the expression of maturation markers (Fig. 5). In contrast to the purified $\mathrm{nDC}$ cultures the inhibitory effect of vemurafenib on pDC maturation is less pronounced. There was a significant decrease in CD80 expression but there was no effect on CD86 expression (Fig. 5a). The maturation of mDCs on the other hand was hardly hampered by vemurafenib (Fig. 5b). PD-L1 upregulation is still inhibited by vemurafenib, showing that the compound is still active and reaching the cells. Furthermore, analysis of secreted cytokines in response to R848 with or without vemurafenib demonstrated no significant effects (Fig. 5c). Thus, vemurafenib has little to no deleterious effects in total PBMC cultures.

\section{Vemurafenib does not inhibit ex vivo maturation of pDCs and $\mathrm{mDCs}$ isolated from melanoma patients on active treatment}

We isolated PBMCs from three melanoma patients before and after 1 month receiving vemurafenib $960 \mathrm{mg}$ BID. We did not observe any significant change in the frequency of either pDCs or $\mathrm{mDC}$ after one month of vemurafenib treatment (Fig. 6a). We investigated the effect of vemurafenib on $\mathrm{pDC}$ and $\mathrm{mDC}$ maturation by stimulating freshly isolated PBMCs from patients on vemurafenib treatment in vitro with R848. These $\mathrm{pDCs}$ and $\mathrm{mDCs}$ matured normally, indicated by the significant increase in CD80, CD86, PD-L1 and

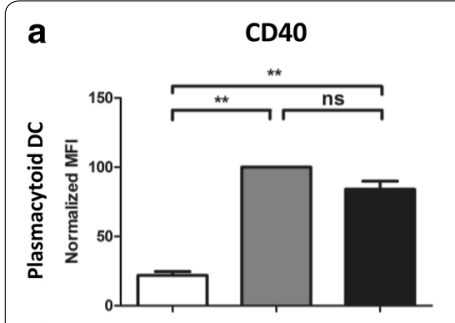

b

CD40

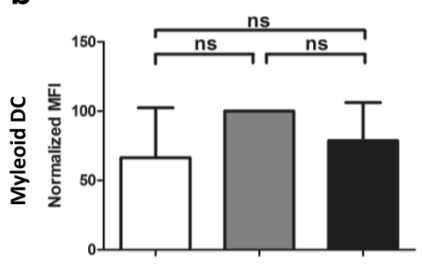

C

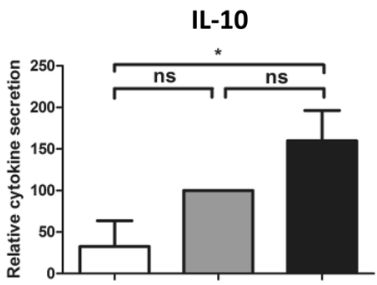

IL-1 $\beta$

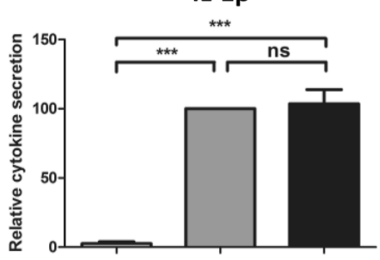

Fig. 5 The presence of immune cells mitigates the inhibitory effects of vemurafenib on DC maturation and cytokine secretion. Freshly isolated PBMCs were cultured ex vivo and activated with R848 in presence or absence of $60 \mu \mathrm{g} / \mathrm{ml}$ vemurafenib. Graphs show the cell surface expression levels of CD80, CD86, CD40, and PD-L1 on pDCs (a) and mDCs (b) after 18 h. c Graphs show the levels of the cytokines IL-10, IL-6, TNFa, IL-1 $\beta$, and IL-5 measured in the supernatant after $18 \mathrm{~h}$. Shown is the mean (+SEM) of four independent experiments ${ }^{*} \mathrm{P}<0.05,{ }^{* *} \mathrm{P}<0.01,{ }^{* * * P}<0.001$ 


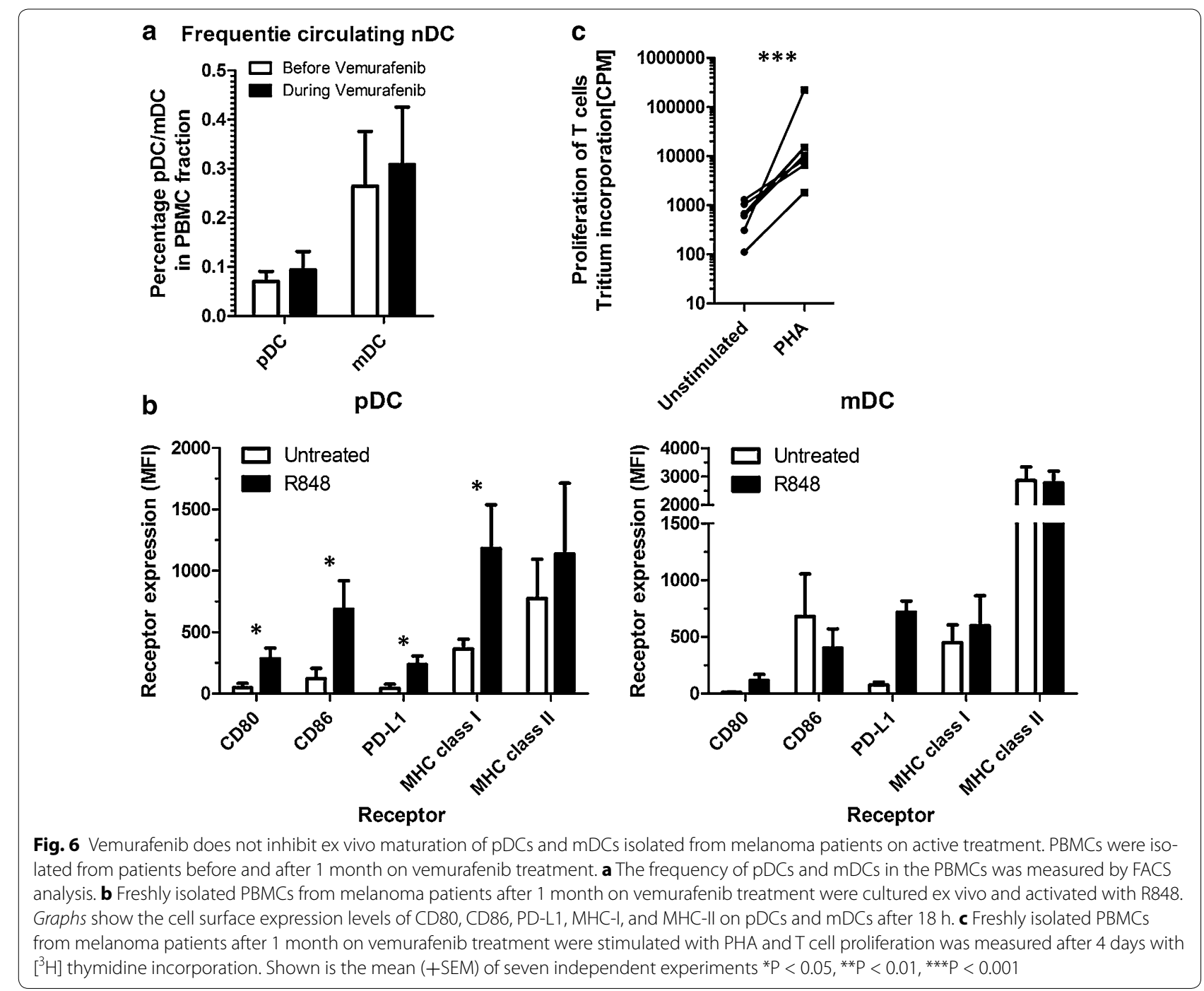

MHC-I (only for pDCs) (Fig. 6b). Additionally, the levels of DC maturation before start of vemurafenib and after one month on vemurafenib were similar (Additional file 4: Fig. S4). Next we investigated the effect of vemurafenib treatment on $\mathrm{T}$ cell activation. We isolated PBMCs from seven Patients receiving vemurafenib and induced non-specific $\mathrm{T}$ cell proliferation by addition of PHA, which led to significant $\mathrm{T}$ cell proliferation in all cases (Fig. 6c). From a subset of these patients we also compared $\mathrm{T}$ cell proliferation before and 1 month after start of vemurafenib and saw no differences (Additional file 4: Fig. S4) From these data, we can conclude that vemurafenib does not negatively affect the frequency of nDC subsets in the blood, nor affects their maturation and also has no negative effects on $\mathrm{T}$ cell function.

\section{Discussion}

Approximately $50 \%$ of melanoma tumors have an activating mutation in BRAF V600 $[8,9]$. This has led to the development of specific BRAF inhibitors such as vemurafenib and dabrafenib. Despite the rapid and impressive response rates, treatment with vemurafenib is limited due to the short duration of the response caused by rapid onset of resistance $[2,12,36]$. Other therapeutic opportunities in order to improve the efficacy of vemurafenib might be found in the combination with DC vaccination. Currently, DC vaccination faces the opposite set of challenges compared to vemurafenib. Many patients do not respond to initial treatment, possibly due to the enormous challenge of fighting a high tumor load in late stage patients. However, the patients that do respond have long-lasting tumor control with life expectancy measured 
in years instead of months $[17,34,37,38]$. This provides the tantalizing possibility of combining vemurafenib with DC vaccination. Vemurafenib treatment could lead to a rapid decrease in tumor load, giving the immune system enough time to mount an effective anti-tumor response that can eliminate residual tumor cells and vemurafenib resistant tumor cells.

Here, we performed an exploratory preclinical, ex vivo, study on the feasibility of combining vemurafenib treatment with DC vaccination; specifically, we studied the potential off-target effects of vemurafenib on human natural blood DC subsets, which appear to be the superior vehicles for DC vaccination [17, 28, 39]. We found that the availability of vemurafenib is a crucial component in testing its toxicity. In this study, we used the concentration of vemurafenib, which is found at steady state pharmacokinetics in patients undergoing treatment [31, 40]. This is in contrast to other studies that have looked at the toxicity of vemurafenib on immune cells, including lymphocytes and monocyte-derived DCs using very low concentrations of vemurafenib $(0.5-5 \mu \mathrm{g} / \mathrm{ml})$ [20, 41]. We reasoned that our chosen concentration was the amount of vemurafenib that these blood-borne DCs would be exposed to. Initial experiments showed that this concentration of $60 \mu \mathrm{g} / \mathrm{ml}$ was toxic for pDCs and mDCs. Increased levels of human serum relieved this toxicity, which could be attributed to the fact that the majority of vemurafenib in the blood stream (>99\%) is protein bound, thus reducing the concentration of free vemurafenib [42]. These results already illustrate that it is very difficult to recreate the correct physiological contexture when doing in vitro experiments and that one should always exercise caution when drawing conclusions. So at this concentration vemurafenib had no effect on $\mathrm{nDC}$ viability, but we did observe a clear and significant inhibition of DC maturation and cytokine secretion. However, when replicating this experiment using the whole PBMC fraction instead of the purified $\mathrm{nDC}$ subsets, we observed that vemurafenib had no effect on DC maturation, or cytokine secretion by the PBMCs. This could again be attributed to vemurafenib availability and thus the limited uptake of vemurafenib by the pDCs and mDCs. Finally, we studied pDCs and $\mathrm{mDCs}$ in PBMC fraction obtained from patients before and after 1 month of vemurafenib treatment. Likewise, DCs were not hampered in their ability to mature. Taken together our results clearly show no sign of negative effects of vemurafenib on DC subsets of patients undergoing treatment. DC frequencies remain the same during treatment and also their function and maturation is unaffected. In all, the combination of vemurafenib with $D C$ vaccination seems feasible.

One major concern with combination regimens is the increased risk of toxicity. Indeed the combination of vemurafenib with ipilimumab was unsuccessful because of increased hepatotoxicity. Currently, a phase II study evaluating the safety and benefit of sequential treatment with vemurafenib and ipilimumab is ongoing (NCT01673854) which is an approach to potentially decrease toxicity while gaining activity. The PD-1 receptor is another inhibitory receptor on T-cells that functions as an immune checkpoint $[43,44]$. Monoclonal antibodies that block PD-1, or its ligand PD-L1 (which is expressed on tumor cells) have demonstrated excellent clinical activity in patients with metastatic melanoma, while generally being less toxic than ipilimumab $[5-7,45]$. One multi-modality approach would be the combination of vemurafenib with PD-1 or PD-L1 blockade. The combination vemurafenib and PD-L1 blockade is currently under investigation (NCT01656642). In light of our results, we would propose the combination of vemurafenib with $D C$ vaccination. An increase in toxicity due to this combination treatment is not foreseen, as the toxicity level of DC vaccination on its own, is low consisting mostly of flu-like symptoms (Grade $1-2$ toxicity) $[16,17,46,47]$. Other interesting targeted therapies are dabrafenib, trametinib, or the combination of dabrafenib and trametinib and combining those with DC vaccination. Indeed the combination of dabrafenib and trametinib seems to be more effective while not having a higher toxicity profile than vemurafenib monotherapy [27], though for the moment vemurafenib is still first line therapeutic option.

Lastly, in biopsies of melanoma metastases, an increased $\mathrm{T}$ cell infiltration after vemurafenib treatment was seen. Furthermore, a correlation between CD8 infiltration and clinical response was observed [22]. This again indicates no negative effects of vemurafenib on tumor-specific immune responses. Indeed the increased expression of tumor associated antigens by tumor cells after exposure to vemurafenib might even synergize with the activation of tumor-specific $\mathrm{T}$ cells by the DC vaccine [23], making this combination all the more attractive.

\section{Conclusion}

Our data shows that vemurafenib does not inhibit the functionality of naturally circulating DC subsets in the context of a complete immune system. Additionally, we show that $\mathrm{pDCs}$ and $\mathrm{mDCs}$ isolated from metastatic melanoma patients treated with vemurafenib display normal functionality. Given the extremely mild toxicity profile of DC vaccination, our results pave the way for a combinatorial treatment strategy consisting of combining vemurafenib with $\mathrm{pDC}$ and/or $\mathrm{mDC}$ vaccination. This protocol would combine the best of both worlds, rapid and efficient tumor debulking by vemurafenib with long lasting anti-tumor immune responses induced by DC vaccination. 


\section{Additional files}

Additional file 1: Fig S1. Freshly isolated pDCs were cultured ex vivo and activated with R848 in presence or absence of increasing concentration of vemurafenib. Graphs show the levels IFNa measured in the supernatant after $18 \mathrm{~h}$. Shown is the mean (+SEM) of three independent experiments.

Additional file 2: Fig S2. Indicated immune cell subsets were cultured in increasing concentrations of vemurafenib and cell viability was determined after $24 \mathrm{~h}$ by FACS analysis. Shown is the mean (+SEM) of three independent experiments.

Additional file 3: Fig S3. Vemurafenib has minimal negative effect on gp100-specific T cell priming. pDCs and mDCs from a HLA-A2.1 + donor were loaded with different concentrations of a melanoma-specific peptide $\left(\mathrm{gp} 1_{280: 288}\right)$ or irrelevant peptide (tyrosinase ${ }_{369: 376}$ ) in the presence of R848 with/without vemurafenib and $\mathrm{gp} 100_{280: 288}$ specific T cells. Graphs show the cell surface expression levels of CD25 after overnight co-culture. Shown is the mean (+SEM) of three independent experiments.

Additional file 4: Fig S4. (A) PHA induced T cell proliferation before and after 1 month on vemurafenib treatment. (B) Freshly isolated PBMCs from melanoma patients before and after one month on vemurafenib treatment were cultured ex vivo and activated with R848. Graphs show the cell surface expression levels of CD80, CD86, PD-L1, MHC-I, and MHC-II on pDCs and mDCs after $18 \mathrm{~h}$

\section{Authors' contributions}

JT, NE, CGF, IJMV and SVH conceived and planned experiments. VD, NH, SB, JT, SVH performed experiments. RK, HW, SB, WG provided clinical samples and patient information. JT and SVH wrote the article. All authors read and approved the final manuscript.

\section{Author details}

${ }^{1}$ Department of Tumor Immunology, Radboud Institute for Molecular Life Sciences, Radboud University Medical Center, 6500 HB Nijmegen, The Netherlands. ${ }^{2}$ Department of Medical Oncology, Radboud University Medical Center Nijmegen, The Netherlands. ${ }^{3}$ Department of Pharmacy, Radboud University Medical Center, Nijmegen, The Netherlands.

\section{Acknowledgements}

We thank Dirk van Rens for all the help and support in acquiring clinical samples. This work was supported by Grants from the Netherlands Organization for Scientific Research (NWO Veni Grant 863.13.024 to JT) and the Dutch Cancer Society (KUN2009-4402), a Grant from the Dutch Cancer Society and Alpe deHuZes foundation to SVH (KUN2013-5958). CGF received an NWO Spinoza award and ERC Advance Grant PATHFINDER (269019). IJMdV received NWO Vici Grant 918.14.655.

\section{Competing interests}

The authors declare that they have no competing interests.

Received: 10 February 2016 Accepted: 30 March 2016

Published online: 14 April 2016

\section{References}

1. Miller AJ, Mihm MC Jr. Melanoma. N Engl J Med. 2006;355:51-65.

2. Chapman PB, Hauschild A, Robert C, Haanen JB, Ascierto P, Larkin J, Dummer R, Garbe C, Testori A, Maio M, et al. Improved survival with vemurafenib in melanoma with BRAF V600E mutation. N Engl J Med. 2011;364:2507-16.

3. Flaherty KT, Puzanov I, Kim KB, Ribas A, McArthur GA, Sosman JA, O'Dwyer PJ, Lee RJ, Grippo JF, Nolop K, Chapman PB. Inhibition of mutated, activated BRAF in metastatic melanoma. N Engl J Med. 2010;363:809-19.
4. Hodi FS, O'Day SJ, McDermott DF, Weber RW, Sosman JA, Haanen JB, Gonzalez R, Robert C, Schadendorf D, Hassel JC, et al. Improved survival with ipilimumab in patients with metastatic melanoma. N Engl J Med. 2010;363:711-23.

5. Larkin J, Chiarion-Sileni V, Gonzalez R, Grob JJ, Cowey CL, Lao CD, Schadendorf D, Dummer R, Smylie M, Rutkowski P, et al. Combined nivolumab and ipilimumab or monotherapy in untreated melanoma. N Engl J Med. 2015;373:23-34

6. Robert C, Schachter J, Long GV, Arance A, Grob JJ, Mortier L, Daud A, Carlino MS, McNeil C, Lotem M, et al. Pembrolizumab versus ipilimumab in advanced melanoma. N Engl J Med. 2015;372:2521-32.

7. Wolchok JD, Kluger H, Callahan MK, Postow MA, Rizvi NA, Lesokhin AM, Segal NH, Ariyan CE, Gordon RA, Reed K, et al. Nivolumab plus ipilimumab in advanced melanoma. N Engl J Med. 2013;369:122-33.

8. Jakob JA, Bassett RL Jr, Ng CS, Curry JL, Joseph RW, Alvarado GC, Rohlfs ML, Richard J, Gershenwald JE, Kim KB, et al. NRAS mutation status is an independent prognostic factor in metastatic melanoma. Cancer. 2012;118:4014-23.

9. Davies H, Bignell GR, Cox C, Stephens P, Edkins S, Clegg S, Teague J, Woffendin $\mathrm{H}$, Garnett MJ, Bottomley W, et al. Mutations of the BRAF gene in human cancer. Nature. 2002;417:949-54.

10. Gray-Schopfer V, Wellbrock C, Marais R. Melanoma biology and new targeted therapy. Nature. 2007;445:851-7.

11. Sloot S, Fedorenko IV, Smalley KS, Gibney GT. Long-term effects of BRAF inhibitors in melanoma treatment: friend or foe? Expert Opin Pharmacother. 2014;15:589-92.

12. Sosman JA, Kim KB, Schuchter L, Gonzalez R, Pavlick AC, Weber JS, McArthur GA, Hutson TE, Moschos SJ, Flaherty KT, et al. Survival in BRAF V600-mutant advanced melanoma treated with vemurafenib. N Engl J Med. 2012;366:707-14.

13. Thakur MD, Salangsang F, Landman AS, Sellers WR, Pryer NK, Levesque MP, Dummer R, McMahon M, Stuart DD. Modelling vemurafenib resistance in melanoma reveals a strategy to forestall drug resistance. Nature. 2013;494:251-5

14. Couzin-Frankel J. Breakthrough of the year 2013. Cancer Immunother Sci. 2013;342:1432-3

15. Vasaturo A, Di Blasio S, Peeters DG, de Koning CC, de Vries JM, Figdor CG, Hato SV. Clinical implications of Co-inhibitory molecule expression in the tumor microenvironment for DC vaccination: a game of stop and go. Front Immunol. 2013:4:417.

16. Bol KF, Figdor CG, Aarntzen EH, Welzen ME, van Rossum MM, Blokx WA, van de Rakt MW, Scharenborg NM, de Boer AJ, Pots JM, et al. Intranodal vaccination with mRNA-optimized dendritic cells in metastatic melanoma patients. Oncoimmunology. 2015;4:e1019197.

17. Tel J, Aarntzen EH, Baba T, Schreibelt G, Schulte BM, Benitez-Ribas D, Boerman OC, Croockewit S, Oyen WJ, van Rossum M, et al. Natural human plasmacytoid dendritic cells induce antigen-specific T-cell responses in melanoma patients. Cancer Res. 2013;73:1063-75.

18. Schreibelt G, Bol KF, Westdorp H, Wimmers F, Aarntzen EH, Duivemande Boer T, van de Rakt MW, Scharenborg NM, de Boer AJ, Pots JM et al. Effective clinical responses in metastatic melanoma patients after vaccination with primary myeloid dendritic cells. Clin Cancer Res. 2015. doi:10.1158/1078-0432.CCR-15-2205.

19. Robert C, Thomas L, Bondarenko I, O'Day S, Weber J, Garbe C, Lebbe C, Baurain JF, Testori A, Grob JJ, et al. Ipilimumab plus dacarbazine for previously untreated metastatic melanoma. N Engl J Med. 2011;364:2517-26.

20. Comin-Anduix B, Chodon T, Sazegar H, Matsunaga D, Mock S, Jalil J, Escuin-Ordinas H, Chmielowski B, Koya RC, Ribas A. The oncogenic BRAF kinase inhibitor PLX4032/RG7204 does not affect the viability or function of human lymphocytes across a wide range of concentrations. Clin Cancer Res. 2010;16:6040-8.

21. Liu C, Peng W, Xu C, Lou Y, Zhang M, Wargo JA, Chen JQ, Li HS, Watowich SS, Yang $Y$, et al. BRAF inhibition increases tumor infiltration by $T$ cells and enhances the antitumor activity of adoptive immunotherapy in mice. Clin Cancer Res. 2013;19:393-403.

22. Wilmott JS, Long GV, Howle JR, Haydu LE, Sharma RN, Thompson JF, Kefford RF, Hersey P, Scolyer RA. Selective BRAF inhibitors induce marked T-cell infiltration into human metastatic melanoma. Clin Cancer Res. 2012;18:1386-94.

23. Boni A, Cogdill AP, Dang P, Udayakumar D, Njauw CN, Sloss CM, Ferrone CR, Flaherty KT, Lawrence DP, Fisher DE, et al. Selective BRAFV600E 
inhibition enhances T-cell recognition of melanoma without affecting lymphocyte function. Cancer Res. 2010;70:5213-9.

24. Ribas A, Hodi FS, Callahan M, Konto C, Wolchok J. Hepatotoxicity with combination of vemurafenib and ipilimumab. N Engl J Med. 2013;368:1365-6.

25. Hu-Lieskovan S, Mok S, Moreno BH, Tsoi J, Robert L, Goedert L, Pinheiro EM, Koya RC, Graeber TG, Comin-Anduix B, Ribas A. Improved antitumor activity of immunotherapy with BRAF and MEK inhibitors in BRAF(V600E) melanoma. Sci Transl Med. 2015;7:279.

26. Minor DR, Puzanov I, Callahan MK, Hug BA, Hoos A. Severe gastrointestinal toxicity with administration of trametinib in combination with dabrafenib and ipilimumab. Pigment Cell Melanoma Res. 2015;28:611-2.

27. Robert C, Karaszewska B, Schachter J, Rutkowski P, Mackiewicz A, Stroiakovski D, Lichinitser M, Dummer R, Grange F, Mortier L, et al. Improved overall survival in melanoma with combined dabrafenib and trametinib. N Engl J Med. 2015;372:30-9.

28. Bol KF, Tel J, de Vries IJ, Figdor CG. Naturally circulating dendritic cells to vaccinate cancer patients. Oncoimmunology. 2013;2:e23431.

29. Tel J, Hato SV, Torensma R, Buschow SI, Figdor CG, Lesterhuis WJ, de Vries IJ. The chemotherapeutic drug oxaliplatin differentially affects blood DC function dependent on environmental cues. Cancer Immunol Immunother. 2012;61:1101-11.

30. Sondergaard JN, Nazarian R, Wang Q, Guo D, Hsueh T, Mok S, Sazegar H, MacConaill LE, Barretina JG, Kehoe SM, et al. Differential sensitivity of melanoma cell lines with BRAFV600E mutation to the specific Raf inhibitor PLX4032. J Transl Med. 2010;8:39.

31. Grippo JF, Zhang W, Heinzmann D, Yang KH, Wong J, Joe AK, Munster P, Sarapa N, Daud A. A phase I, randomized, open-label study of the multiple-dose pharmacokinetics of vemurafenib in patients with BRAF V600E mutation-positive metastatic melanoma. Cancer Chemother Pharmacol. 2014;73:103-11.

32. Falchook GS, Long GV, Kurzrock R, Kim KB, Arkenau HT, Brown MP, Hamid O, Infante JR, Millward M, Pavlick A, et al. Dose selection, pharmacokinetics, and pharmacodynamics of BRAF inhibitor dabrafenib (GSK2118436). Clin Cancer Res. 2014;20:4449-58.

33. Infante JR, Fecher LA, Falchook GS, Nallapareddy S, Gordon MS, Becerra C, DeMarini DJ, Cox DS, Xu Y, Morris SR, et al. Safety, pharmacokinetic, pharmacodynamic, and efficacy data for the oral MEK inhibitor trametinib: a phase 1 dose-escalation trial. Lancet Oncol. 2012;13:773-81.

34. Aarntzen EH, De Vries IJ, Lesterhuis WJ, Schuurhuis D, Jacobs JF, Bol K, Schreibelt G, Mus R, De Wilt JH, Haanen JB, et al. Targeting CD4(+) T-helper cells improves the induction of antitumor responses in dendritic cell-based vaccination. Cancer Res. 2013;73:19-29.

35. Simon T, Fonteneau JF, Gregoire M. Requirement of tumor-associated antigen-specific CD4+ T cells for an efficient dendritic cell vaccine in antitumor immunotherapy. Immunotherapy. 2013;5:565-7.
36. Straussman R, Morikawa T, Shee K, Barzily-Rokni M, Qian ZR, Du J, Davis A, Mongare MM, Gould J, Frederick DT, et al. Tumour micro-environment elicits innate resistance to RAF inhibitors through HGF secretion. Nature. 2012;487:500-4.

37. Banchereau J, Ueno H, Dhodapkar M, Connolly J, Finholt JP, Klechevsky E, Blanck JP, Johnston DA, Palucka AK, Fay J. Immune and clinical outcomes in patients with stage IV melanoma vaccinated with peptide-pulsed dendritic cells derived from CD34+ progenitors and activated with type I interferon. J Immunother. 2005;28:505-16.

38. Wilgenhof S, Van Nuffel AM, Corthals J, Heirman C, Tuyaerts S, Benteyn D, De Coninck A, Van Riet I, Verfaillie G, Vandeloo J, et al. Therapeutic vaccination with an autologous mRNA electroporated dendritic cell vaccine in patients with advanced melanoma. J Immunother. 2011;34:448-56.

39. Wimmers F, Schreibelt G, Skold AE, Figdor CG, De Vries IJ. Paradigm shift in dendritic cell-based immunotherapy: from in vitro generated monocytederived DCs to naturally circulating DC subsets. Front Immunol. 2014;5:165.

40. Funck-Brentano E, Alvarez JC, Longvert C, Abe E, Beauchet A, FunckBrentano C, Saiag P. Plasma vemurafenib concentrations in advanced BRAFV600mut melanoma patients: impact on tumour response and tolerancedagger. Ann Oncol. 2015;26(7):1470-5. doi:10.1093/annonc/ mdv189.

41. Ott PA, Henry T, Baranda SJ, Frleta D, Manches O, Bogunovic D, Bhardwaj N. Inhibition of both BRAF and MEK in BRAF(V600E) mutant melanoma restores compromised dendritic cell (DC) function while having differential direct effects on DC properties. Cancer Immunol Immunother. 2013;62:811-22.

42. Sharma A, Shah SR, Illum H, Dowell J. Vemurafenib: targeted inhibition of mutated BRAF for treatment of advanced melanoma and its potential in other malignancies. Drugs. 2012;72:2207-22.

43. Okazaki T, Chikuma S, Iwai Y, Fagarasan S, Honjo T. A rheostat for immune responses: the unique properties of PD-1 and their advantages for clinical application. Nat Immunol. 2013;14:1212-8.

44. Pardoll DM. The blockade of immune checkpoints in cancer immunotherapy. Nat Rev Cancer. 2012;12:252-64.

45. Hamid O, Robert C, Daud A, Hodi FS, Hwu WJ, Kefford R, Wolchok JD, Hersey P, Joseph RW, Weber JS, et al. Safety and tumor responses with lambrolizumab (anti-PD-1) in melanoma. N Engl J Med. 2013;369:134-44.

46. Kamigaki T, Matsuda E, Okada S, Naitoh K, Kondo T, Ibe H, Maekawa $R$, Goto S. Prospective evaluation of safety of immune-cell therapy for patients with various types of advanced cancer. Anticancer Res. 2014;34:4601-7.

47. Lesterhuis WJ, Schreibelt G, Scharenborg NM, Brouwer HM, Gerritsen MJ, Croockewit S, Coulie PG, Torensma R, Adema GJ, Figdor CG, et al. Wild-type and modified gp100 peptide-pulsed dendritic cell vaccination of advanced melanoma patients can lead to long-term clinical responses independent of the peptide used. Cancer Immunol Immunother. 2011;60:249-60.

\section{Submit your next manuscript to BioMed Central and we will help you at every step:}

- We accept pre-submission inquiries

- Our selector tool helps you to find the most relevant journal

- We provide round the clock customer support

- Convenient online submission

- Thorough peer review

- Inclusion in PubMed and all major indexing services

- Maximum visibility for your research

Submit your manuscript at www.biomedcentral.com/submit

\section{Biomed Central}

